\title{
Autoimmune premature ovarian failure
}

\author{
Beata Komorowska \\ Outpatient Clinic, Teaching Hospital No. 1 in Lublin, Poland
}

\begin{abstract}
Premature ovarian failure (POF), also termed as primary ovarian insufficiency (POI), is a highly heterogenous condition affecting $0.5-3.0 \%$ of women in childbearing age. These young women comprise quite a formidable group with unique physical and psychological needs that require special attention. Premature ovarian senescence (POS) in all of its forms evolves insidiously as a basically asymptomatic process, leading to complete loss of ovarian function, and POI/POF diagnoses are currently made at relatively late stages. Well-known and well-documented risk factors exist, and the presence or suspicion of autoimmune disorder should be regarded as an important one. Premature ovarian failure is to some degree predictable in its occurrence and should be considered while encountering young women with loss of menstrual regularity, especially when there is a concomitant dysfunction in the immune system.
\end{abstract}

Key words: premature ovarian failure, primary ovarian insufficiency, autoimmune disorders, anti-ovarian antibodies, adrenal insufficiency.

\section{Introduction}

Premature ovarian failure (POF) is the occurrence of hypergonadotrophic hypoestrogenic amenorrhoea in women under the age of 40 years. The median age of menopause in western populations of women is approximately 51 years. While very late (i.e. after 55 years) menopause is rather uncommon, a minority of women experience cessation of ovarian function at or prior to 45 years of age. By established convention, menopause that occurs at ages 40-45 years is considered "early" and occurs in about $5 \%$ of women. The term premature ovarian failure is reserved for the approximately $1 \%$ of women who experience hypergonadotrophic amenorrhoea prior to 40 years of age [1]. Premature ovarian failure represents the end stage of a variety of disorders that result in the loss of ovarian follicles. Depending upon the age at diagnosis, the probability of a genetic, autoimmune, or idiopathic cause will be more or less likely.

Increased incidence of gonadal failure observed in patients with autoimmune disorders such as primary adrenal insufficiency (Addison's disease) or autoimmune thyroid disease (Grave's or Hashimoto disease) directed research in the 1960 s and 1970 s toward potential autoimmune aetiologies of POF. Estimates may differ, but about $5-30 \%$ of POF cases have an autoimmune aetiology [2-4].

Untreated early ovarian failure increases the risk of osteoporosis, cardiovascular disease, dementia, cognitive decline, and Parkinsonism [5].

\section{Terminology}

Premature ovarian insufficiency (POI) was recognised in the 1930s in amenorrhoeic young women with elevated urinary gonadotropins [6] and was first described by Fuller Albright in 1942 as menopausal histological changes in the ovaries in conjunction with adrenal insufficiency [7, 8].

The term POF appeared in the 1960s and was defined as at least four months of amenorrhoea associated with elevated follicle stimulating hormone (FSH) levels in a woman under 40 years of age [9]. Since this definition excludes a number of patients with clinically significant ovarian dysfunction, experiencing a long and unpredictable course of the disease but not meeting the above diagnostic criteria, the term POI has returned to more accurately reflect the clinical spectrum of the problem [10].

Other terms used for this condition include primary ovarian dysfunction, primary ovarian failure, and hypergonadotrophic hypogonadism, as well as the misnomer: gonadal dysgenesis. The condition is considered to be present when a woman who is less than 40 years old has had amenorrhoea for four months or more, with two serum FSH levels (obtained at least one month apart) in the menopausal range [10]. The condition is different from menopause because there is intermittent and unpredictable ovarian function in approximately $50 \%$ of cases, spontaneous ovulations in $20 \%$, and about $5-10 \%$ of women conceive and deliver a child 
after the diagnosis. Thus, the term "primary ovarian insufficiency," as originally suggested by Albright, meets the need to describe a continuum of impaired ovarian function rather than a dichotomous state. This term may also be less stigmatising than the terms that were used previously [11].

The most common known causes of POI are:

- iatrogenic; following surgery, radiotherapy, or chemotherapy,

- chromosomal and genetic aberrations,

- autoimmune ovarian damage,

- environmental factors (viruses, chemical agents, radiation, etc.),

- metabolic (diabetes type 1, galactosaemia, 17-OH deficiency, 21-OH deficiency, etc.),

- endometriosis,

- polycystic ovary syndrome (PCOS).

\section{Immunology}

The human ovary is commonly the target of an autoimmune attack leading to ovarian dysfunction, which can be manifested as POF, PCOS, unexplained infertility, or endometriosis [12]. In the case of POF, the evidence for an autoimmune aetiology is based on the presence of lymphocytic oophoritis, association with other autoimmune disorders, and autoantibodies to ovarian antigens, which has been clearly documented in numerous studies $[11,13]$. The development of POF has been associated with autoimmune reactions to ovarian tissue in more than half of the cases [14]. Autoimmune ovarian damage is caused by the alteration of T-cell subsets and T-cell-mediated injury, increase of autoantibody-producing B-cells and a low number of effector suppressor/cytotoxic lymphocytes, and a decrease in the number and activity of natural killer cells. Autoimmune POF can be associated with other non-endocrine and endocrine diseases [15].

Autoimmune lymphocytic oophoritis was first described in the presence of Addison's disease and adrenal immunity [3]. It is characterised by mononuclear inflammatory cell (plasma B and T-cells) infiltrate in the theca cells of growing follicles, with well-established sparing of early stage (primordial) follicles. This distinctive pathophysiologic process presents the theoretical possibility of developing immunosuppressive treatments that could restore fertility $[4,16]$.

Much more frequently, ovaries appear to be subject to a still poorly defined autoimmune attack associated with thyroid autoimmunity, anti-adrenal autoimmunity, and other, often non-organ-specific, autoimmune responses [3].

The increasing recognition of the $X$ chromosome as an "autoimmune chromosome" also explains the very high prevalence of associated autoimmunity with Turner syndrome [13].
About $20 \%$ of POF patients have been previously diagnosed with other concomitant autoimmune diseases. The most common of them are disorders of the thyroid, adrenals, and pancreas. Approximately $10 \%$ of women afflicted with Addison's disease develop POF. Probably the best-defined form of autoimmune-associated POF occurs with one of the four known autoimmune polyglandular syndromes (APS), so-called APS-1, also known as the polyendocrinopathy candidiasis ectodermal dystrophy or Whitaker syndrome. It is caused by a mutation in the autoimmune regulator (AIRE) gene [15, 17]. This gene is of importance in the thymus, where it regulates self-tolerance from T-cell attacks. Mutations in the gene, therefore, has been associated with attacks against "self" [18]. As a result $45-60 \%$ of female subjects develop POF. By comparison, testicular failure is relatively rare afflicting about $2 \%$ of male carriers.

APS-2 is a more common condition and includes a primary adrenal insufficiency, autoimmune thyroid disease, type 1 diabetes mellitus, and others (celiac disease, myasthenia gravis). It affects 14-20 million people with prominent female predominance. $5-50 \%$ of them have early ovarian or testicular failure [19]. In this syndrome, alleles of human leukocyte antigens (HLAs) determine the targeting of specific tissues by autoreactive $T$ cells, which leads to organ-specific autoimmunity as a result of this loss of tolerance $[17,18]$.

Several other autoimmune disorders have been associated with POF; hypothyroidism is the most common. Both endocrine (thyroid, hypoparathyroid, diabetes mellitus, hypophysitis) and non-endocrine disorders (chronic candidiasis, idiophatic thrombocytopaenic purpura, vitiligo, alopecia, autoimmune haemolytic anaemia, pernicious anaemia, systemic lupus erythematosus (SLE), rheumatoid arthritis, Crohn's disease, Sjögren's syndrome, primary biliary cirrhosis, and chronic active hepatitis) have been observed in association with autoimmune POF [20, 21].

Anti-oocyte antibodies were identified in 1966, and this was also one of the first descriptions of anti-ovarian autoimmunity [22]. Since then the search for anti-ovarian antibodies (AOA) has been undertaken in numerous studies, especially in patients with POI; however, their results are still conflicting, particularly due to differences in laboratory methods as well as many ovarian components being potential antigens [12].

An investigation of anti-ovarian autoimmune reactions and autoantibodies may be severely hampered by the fact that POF represents an end-stage of the disease. By the time a woman is diagnosed, she has exhausted her follicular supply and, presumably, also the target antigen for the autoimmune attack on her ovary. Thus, the autoimmunity causal of POI can be difficult to detect retrospectively. Regardless of that, a high prevalence of anti-ovarian antibodies (AOA) (30-67\%) and other organ- and non-organ-specific autoantibodies has been observed in patients with POI [14, 23, 24]. 
Some antibodies in the pool of AOA are thought to associate with a direct action on ovarian tissue, whereas others have no such effects, similarly to autoantibodies in other autoimmune diseases [25]. Therefore, it is possible that several different antigens are involved in ovarian autoimmunity because both ovarian cellular and zona pellucida/oocyte antibodies have been reported [14, 23].

There are a considerable number of known antigens identified as molecular targets of AOA; steroidogenic enzymes such as: $17 \alpha$-hydroxylase, desmolase (P450side chain cleavage), 3 $\beta$-hydroxysteroid dehydrogenase, and 21-hydroxylase [14, 24]. Human heat shock protein $90-\beta$ (strongly induced during chlamydial infections) and anti- $\alpha$-enolase have also been identified as unique antigens in anti-ovarian autoimmunity associated with POI and infertility [14].

Corpus luteum antibodies are present in $22 \%$ of patients with SLE. The presence of such antibodies in the patients who were all $<40$ years of age correlates with elevated serum FSH levels. Therefore, anti-corpus luteum antibodies could represent the first stage of altered ovarian function in SLE patients [26].

Gonadotrophin receptors have also been investigated as potential autoantibody targets. The majority of anti-ovarian autoantibodies are directed against the $\beta$-subunit of follicle stimulating hormone (anti-FSH). They might modulate the recognition and binding of FSH to its receptor and might, therefore, have a pathological influence on ovarian function, and there is evidence of an association between their presence and POI [14].

Endometriosis has been labelled an "autoimmune syndrome" because, as like classical autoimmune diseases, it is characterised by polyclonal B-cell activation and production of multiple different autoantibodies. About $40-60 \%$ of patients with endometriosis have elevated autoantibody titres when tested against a panel of autoantigens. They often possess specific anti-endometrial antibodies, but also AOA, antinuclear autoantibodies (ANA), smooth muscle autoantibodies (SMA), and antiphospholipid antibodies (APA) [13, 26-28].

There have been case reports linking POF with immunisations via anti-ovarian antibody formation, especially concerning quadrivalent anti-HPV vaccine [29-31]. It has been considered that safety research before and after licensing has inadequate capacity to determine ovarian safety. Since case reports do not and cannot establish causation, further investigation in this area is still required [30].

\section{Management}

\section{Diagnosis}

Presenting symptoms: amenorrhoea, oligomenorrhoea, infertility.
Physical examination is unremarkable in the majority of cases, but it may reveal evidence of an associated disorder such as hypotension, decreased axillary and pubic hair, hyperpigmentation or vitiligo (which is associated with autoimmune adrenal insufficiency), thyroid enlargement, exophthalmos, bradycardia or tachycardia, and cold-and-dry or soft-and-warm skin (indicative of thyroid pathology). Other findings associated with the presence of autoimmune diseases may include vitiligo (often associated with thyroid and adrenal autoimmunity), premature greying of hair (in thyroid diseases), nail dystrophy and mucocutaneous candidiasis (in autoimmune polyglandular syndrome type 1), and alopecia areata and malar rash (in lupus) [19, 20, 32].

Pelvic examination usually reveals atrophic vaginitis. However, some women have intermittent follicular function and produce enough oestradiol for adequate oestrogenisation of the vaginal mucosa. In most of the cases the ovaries are small and barely palpable. Enlarged ovaries could be found occasionally, as in some cases of immune oophoritis [33]. Actually in autoimmune oophoritis the presence of large multifollicular ovaries combined with elevated serum luteinising hormone (LH) levels could be mistaken for PCOS. However, in such cases there will be no androgen excess, but rather androgen deficiency related to impaired thecal and adrenal production of androgens [16, 34].

Ultrasound has limited value as a diagnostic tool [35]; however, it is useful in establishing the presence of follicular activity [36] and ruling out other amenorrhea causes [37], so it should be performed in every case of menstrual irregularities.

A small group of women with POF present with large luteinised follicular cyst similar to that reported in 17-20 desmolase deficiency. Concomitant laboratory findings suggest theca cell destruction with preserved granulosa cell function characteristic of autoimmune oophoritis $[18,38]$.

Laboratory tests for the initial evaluation of patients presenting with secondary amenorrhoea, after pregnancy have been excluded, including measurement of serum $\mathrm{FSH}$, estradiol, thyroid-stimulating hormone (TSH), and serum prolactin. If FSH is in the menopausal range, the test should be repeated after a 4-6-week interval along with estradiol, to confirm hypogonadism [37].

Progesterone challenge test may have some diagnostic value but could be misleading in the presence of intermittent ovarian function and should not be used instead of measurement of serum hormone levels.

When fertility is desired, ovarian peptides such as anti-Mullerian hormone (AMH) and Inhibin B are useful in evaluation of the follicular reserve $[5,13,39]$.

Recently several research groups demonstrated age-specificity in $\mathrm{AMH}$ levels, providing norms for all premenopausal age groups. This allows more accurate 
diagnosis of POF and offers a "screening" tool and the possibility of assessing prospective risk of developing POF in the future for very young women with known risk factors [13].

\section{Anti-oocyte autoantibodies}

In animal studies using thymectomised neonatal mice AOA titres correlated with the degree and progression of oophoritis $[40,41]$. A decline in the AOA titres correlated with the decline in ovarian function. These results appear promising, but attempts to use AOA titres to screen for autoimmune POF aetiologies have been disappointing. Commercially available tests using ELISA and indirect immunofluorescence techniques produce inconsistent results and high false positive rates [42, 43]

\section{Associated disorders}

About $2-10 \%$ of POF patients have concomitant autoimmune adrenal gland disorders [3]. This may be a clinically apparent disease or a subclinical process with anti-adrenal antibody production, but without adrenal insufficiency. According to some researchers, POF may precede Addison disease by 8-14 years. There is a $50 \%$ risk of the development of adrenal insufficiency in women with adrenal autoimmunity. All patients with primary ovarian insufficiency should be educated regarding the symptoms of adrenal insufficiency and should undergo evaluation of adrenal function if such symptoms develop $[20,32,44]$. The best markers of occult autoimmune adrenal insufficiency are circulating 21-hydroxylase antibodies [20].

Thyroid autoimmune disease, most commonly Hashimoto's thyroiditis, is present in $14-27 \%$ of women at initial diagnosis [11]. It is reasonable to measure thyrotropin levels and test for the presence of thyroid peroxidase antibodies.

All women with the above-mentioned problems will require referral to an endocrinologist for additional evaluation and long-term follow-up.

\section{Treatment}

Management should be directed at symptom resolution and bone protection, but most importantly should include psychosocial support for women facing this devastating diagnosis [10].

All POF patients should receive cyclical hormonal therapy with oestrogens and progestins. However, oestrogens may exacerbate some of the autoimmune diseases SLE, so all such patients should be monitored closely at the installation of hormonal therapy.

Androgens may be considered in case of chronic fatigue, lack of libido, and poor wellbeing despite ade- quate oestrogen replacement and when depression has been ruled out or adequately treated [34]. This therapy should be used with great caution and for short periods of time until more data are available.

Gonadotropin therapy is not advised since it may exacerbate autoimmune POF and increase follicle turnover lowering in effect follicular reserve.

Use of prednisone or dexamethasone to suppress AOA is not indicated clinically because it poses a risk of osteoporosis and cause iatrogenic Cushing's syndrome [45].

\section{Follow-up}

Patients with ovarian failure should have annual follow-up visits to monitor their hormonal therapy.

Symptoms and signs of thyroid disease and adrenal insufficiency should be sought during the annual follow-up visits.

TSH levels should be checked every 3-5 years (every year if antiperoxidase antibody test is positive).

If adrenal antibodies are detected on initial POI/ POF evaluation, even if all adrenal function tests are normal, the patient is at high risk of developing adrenal insufficiency and should have an ACTH stimulation test repeated every year [11]. Some authors, however, advise caution because the test was designed as a diagnostic tool for symptomatic patients and its use in screening may yield a number of false positive results [46]. Whether women with initially negative adrenal antibody tests continue to carry higher than normal risk for adrenal insufficiency and whether any follow-up tests are justified is less clear. Until enough evidence is acquired, it would be cautious to perform adrenal antibody tests every 3-5 years.

\section{Summary}

A number of clinical and biological features indicate that autoimmunity is involved in several ovarian pathologies such as POF, idiopathic infertility, polycystic ovary syndrome, or endometriosis. Currently treatment options are limited and specific guidelines for the treatment of autoimmune POF are not available. Ongoing research is focused on the development of more accurate diagnostic tools for the determination of the real prevalence of autoimmune aetiology in ovarian disease, detection of concomitant or future associated autoimmune disorders, as well as selection of patients in whom immune-modulating therapy may restore, at least temporarily, ovarian function and fertility.

Precocious and reliable diagnosis of an autoimmune aetiology is particularly important for the young women in pre-procreational stage of life when adjustment of plans for the future and avoidance of additional damaging factors is still possible. 


\section{References}

1. Santoro N. Mechanisms of premature ovarian failure. Ann Endocrinol (Paris) 2003; 64: 87-92.

2. Nelson LM, Bakalov VK. Mechanisms of follicular dysfunction in $46, \mathrm{XX}$ spontaneous premature ovarian failure. Endocrinol Metab Clin North Am 2003; 32: 613-637.

3. Hoek A, Schoemaker J, Drexhage HA. Premature ovarian failure and ovarian autoimmunity. Endocr Rev 1997; 18: 107-134.

4. Silva CA, Yamakami LY, Aikawa NE, et al. Autoimmune primary ovarian insufficiency. Autoimmun Rev 2014; 13: 427-430.

5. Vujovic S, Brincat M, Erel T, et al. EMAS position statement: Managing women with premature ovarian failure. Maturitas 2010; 67: 91-93.

6. Heller CG, Heller EJ. Gonadotropic hormone: urine assays of normally cycling, menopausal, castrated, and estrin treated human females 1. J Clin Invest 1939; 18: 171-178.

7. Bartter FC, Albright F, Forbes AP, et al. The effects of adrenocorticotropic hormone and cortisone in the adrenogenital syndrome associated with congenital adrenal hyperplasia: an attempt to explain and correct its disordered hormonal pattern 12. J Clin Invest 1951; 30: 237-251.

8. Albright F, Smith PH, Fraser R. A syndrome characterized by primary ovarian insufficiency and decreased stature: report of 11 cases with a digression on hormonal control of axillary and pubic hair. Am J Med Sci 1942; 204: 625-648.

9. de Moraes-Ruehsen M, Jones GS. Premature ovarian failure. Fertil Steril 1967; 18: 440-461

10. Welt CK. Primary ovarian insufficiency: a more accurate term for premature ovarian failure. Clin Endocrinol (Oxf) 2008; 68: 499-509.

11. Nelson LM. Clinical practice. Primary ovarian insufficiency. N Engl I Med 2009; 360: 606-614

12. Petríková J, Lazúrová I. Ovarian failure and polycystic ovary syndrome. Autoimmun Rev 2012; 11: A471-A478.

13. Gleicher N, Kushnir VA, Barad DH. Prospectively assessing risk for premature ovarian senescence in young females: a new paradigm. Reprod Biol Endocrinol 2015; 13: 34.

14. Haller-Kikkatalo K, Salumets A, Uibo R. Review on autoimmune reactions in female infertility: antibodies to follicle stimulating hormone Clin Dev Immunol 2012; 2012: 762541

15. Vujovic S. Aetiology of premature ovarian failure. Menopause Int 2009; 15: $72-75$

16. Bakalov VK, Anasti JN, Calis KA, et al. Autoimmune oophoritis as a mechanism of follicular dysfunction in women with 46,XX spontaneous premature ovarian failure. Fertil Steril 2005; 84: 958-965.

17. Michels AW, Gottlieb PA. Autoimmune polyglandular syndromes. Nat Rev Endocrinol 2010; 6:270-277.

18. Eisenbarth GS (ed.). Immunoendocrinology: Scientific and Clinical As pects, Contemporary Endocrinology.: Springer Science and Business Media, New York 2011

19. Ahonen P, Myllärniemi S, Sipilä I, Perheentupa J. Clinical variation of autoimmune polyendocrinopathy-candidiasis-ectodermal dystrophy (APECED) in a series of 68 patients. N Engl J Med 1990; 322: 1829-1836.

20. Betterle C, Dal Pra C, Mantero F, Zanchetta R. Autoimmune adrenal insufficiency and autoimmune polyendocrine syndromes: autoantibodies, autoantigens, and their applicability in diagnosis and disease predic tion. Endocr Rev 2002; 23: 327-364.

21. Ebrahimi M, Akbari Asbagh F. Pathogenesis and causes of premature ovarian failure: an update. Int J Fertil Steril 2011; 5: 54-65.

22. Vallotton MB, Forbes AP. Antibodies to cytoplasm of ova. Lancet (London, England) 1966; 2: 264-265.

23. Kelkar RL, Meherji PK, Kadam SS, et al. Circulating auto-antibodies against the zona pellucida and thyroid microsomal antigen in women with premature ovarian failure. J Reprod Immunol 2005; 66: 53-67.

24. Luborsky JL, Visintin I, Boyers S, et al. Ovarian antibodies detected by immobilized antigen immunoassay in patients with premature ovarian failure. J Clin Endocrinol Metab 1990; 70: 69-75.

25. Luborsky J. Ovarian autoimmune disease and ovarian autoantibodies. J Womens Health Gend Based Med 2002; 11: 585-599.

26. Forges T, Monnier-Barbarino P, Faure GC, Béné MC. Autoimmunity and antigenic targets in ovarian pathology. Hum Reprod Update 2004; 10 : $163-175$.
27. Cordts EB, Santos AA, Peluso C, et al. Risk of premature ovarian failure is associated to the Pvull polymorphism at estrogen receptor gene ESR1. J Assist Reprod Genet 2012; 29: 1421-1425.

28. Haller-Kikkatalo K, Uibo R, Kurg A, Salumets A. The prevalence and phenotypic characteristics of spontaneous premature ovarian failure: a general population registry-based study. Hum Reprod 2015; 30: 1229 1238.

29. Little DT, Ward HR. Premature ovarian failure 3 years after menarche in a 16-year-old girl following human papillomavirus vaccination. BMJ Case Rep 2012; pii: bcr2012006879.

30. Little DT, Ward HR. Adolescent premature ovarian insufficiency following human papillomavirus vaccination: a case series seen in general practice. J Investig Med High Impact Case Rep 2014; 2: 2324709614556129.

31. Colafrancesco S, Perricone C, Tomljenovic L, Shoenfeld Y. Human papilloma virus vaccine and primary ovarian failure: another facet of the autoimmune/inflammatory syndrome induced by adjuvants. Am J Reprod Immunol 2013; 70: 309-316.

32. Betterle C, Volpato M. Adrenal and ovarian autoimmunity. Eur J Endocrinol 1998; 138: 16-25.

33. Lonsdale RN, Roberts PF, Trowell JE. Autoimmune oophoritis associated with polycystic ovaries. Histopathology 1991; 19: 77-81.

34. Kalantaridou SN, Calis KA, Vanderhoof VH, et al. Testosterone deficiency in young women with $46, \mathrm{XX}$ spontaneous premature ovarian failure. Fertil Steril 2006; 86: 1475-1482.

35. Goswami D, Conway GS. Premature ovarian failure. Hum Reprod Update 2005; 11: 391-410.

36. Conway GS, Kaltsas G, Patel A, et al. Characterization of idiopathic premature ovarian failure. Fertil Steril 1996; 65: 337-341.

37. Panay N, Kalu E. Management of premature ovarian failure. Best Pract Res Clin Obstet Gynaecol 2009; 23: 129-140.

38. Biscotti CV, Hart WR, Lucas JG. Cystic ovarian enlargement resulting from autoimmune oophoritis. Obstet Gynecol 1989; 74 (3 Pt 2): 492 495.

39. Skałba P. Anti-Müllerian hormone: plasma levels in women with polycystic ovary syndrome and with premature ovarian failure. Menopause Rev 2011; 3: 232-236.

40. Nair S, Mastorakos G, Raj S, Nelson LM. Murine experimental autoimmune oophoritis develops independently of gonadotropin stimulation and is primarily localized in the stroma and theca. Am J Reprod Immuno 1995; 34: 132-139.

41. Miyake T, Taguchi O, Ikeda H, et al. Acute oocyte loss in experimental autoimmune oophoritis as a possible model of premature ovarian failure. Am J Obstet Gynecol 1988; 158: 186-192.

42. Wheatcroft NJ, Salt C, Milford-Ward A, et al. Identification of ovarian antibodies by immunofluorescence, enzyme-linked immunosorbent assay or immunoblotting in premature ovarian failure. Hum Reprod 1997 12: 2617-2622.

43. Novosad JA, Kalantaridou SN, Tong ZB, Nelson LM. Ovarian antibodies as detected by indirect immunofluorescence are unreliable in the diagnosis of autoimmune premature ovarian failure: a controlled evaluation. BMC Womens Health 2003; 3: 2.

44. Nelson LM, Covington SN, Rebar RW. An update: Spontaneous premature ovarian failure is not an early menopause. Fertil Steril 2005; 83 1327-1332.

45. Kalantaridou SN, Braddock DT, Patronas NJ, Nelson LM. Treatment of autoimmune premature ovarian failure. Hum Reprod 1999; 14: 1777 1782.

46. Bakalov VK, Vanderhoof VH, Bondy CA, Nelson LM. Adrenal antibodies detect asymptomatic auto-immune adrenal insufficiency in young women with spontaneous premature ovarian failure. Hum Reprod 2002; 17: 2096-2100. 